\title{
Transient hydrodynamic finite-size effects in simulations under periodic boundary conditions
}

\author{
Adelchi J. Asta, ${ }^{1}$ Maximilien Levesque, ${ }^{2,3}$ Rodolphe Vuilleumier, ${ }^{2,3}$ and Benjamin Rotenberg ${ }^{1,4}$ \\ ${ }^{1}$ Sorbonne Universités, UPMC Univ Paris 06, CNRS, UMR 8234 PHENIX, 4 Place Jussieu, 75005 Paris, France \\ ${ }^{2}$ Ecole Normale Supérieure, PSL Research University, UPMC Univ Paris 06, CNRS, Département de Chimie, \\ PASTEUR, 24 rue Lhomond, 75005 Paris, France \\ ${ }^{3}$ Sorbonne Universités, UPMC Univ Paris 06, ENS, CNRS, PASTEUR, 75005 Paris, France \\ ${ }^{4}$ Réseau sur le Stockage Electrochimique de l'Energie (RS2E), FR CNRS 3459, France
}

(Received 4 February 2017; published 20 June 2017)

\begin{abstract}
We use lattice-Boltzmann and analytical calculations to investigate transient hydrodynamic finite-size effects induced by the use of periodic boundary conditions. These effects are inevitable in simulations at the molecular, mesoscopic, or continuum levels of description. We analyze the transient response to a local perturbation in the fluid and obtain the local velocity correlation function via linear response theory. This approach is validated by comparing the finite-size effects on the steady-state velocity with the known results for the diffusion coefficient. We next investigate the full time dependence of the local velocity autocorrelation function. We find at long times a crossover between the expected $t^{-3 / 2}$ hydrodynamic tail and an oscillatory exponential decay, and study the scaling with the system size of the crossover time, exponential rate and amplitude, and oscillation frequency. We interpret these results from the analytic solution of the compressible Navier-Stokes equation for the slowest modes, which are set by the system size. The present work not only provides a comprehensive analysis of hydrodynamic finite-size effects in bulk fluids, which arise regardless of the level of description and simulation algorithm, but also establishes the lattice-Boltzmann method as a suitable tool to investigate such effects in general.
\end{abstract}

DOI: 10.1103/PhysRevE.95.061301

It is by now well established that hydrodynamic finite-size effects arise in simulations due to the use of periodic boundary conditions (PBCs). These effects can be understood as the result of spurious hydrodynamic interactions between particles and their periodic images. Following Dünweg and Kremer [1], Yeh and Hummer [2] proposed a complete analysis of the finite-size effect on the diffusion coefficient of fluid particles in a cubic box based on the mobility tensor $\mathbb{T}$ :

$$
\mathbb{D}_{\mathrm{PBC}}=D_{\infty} \mathbb{1}+k_{B} T \lim _{r \rightarrow 0}\left[\mathbb{T}_{\mathrm{PBC}}(\mathbf{r})-\mathbb{T}_{\infty}(\mathbf{r})\right],
$$

with $k_{B}$ Boltzmann's constant and $T$ the temperature and where PBC and subscript $\infty$ denote properties under periodic and unbounded conditions, respectively, while $\mathbb{1}$ is the identity matrix. This results in a finite-size scaling of the diffusion coefficient $D(L)=D_{\infty}-\xi k_{B} T / 6 \pi \eta L$ for a cubic box of size $L$, with $\xi \approx 2.837$ a constant and $\eta$ the fluid viscosity. The same scaling was found independently [3] and has been confirmed in molecular dynamics simulations of simple fluids [2], including several water models [4,5], ionic liquids [6], or more complex fluids such as solutions of star polymers [7]. More recently, the extension to anisotropic boxes was also investigated $[8,9]$ and interpreted in terms of the same hydrodynamic arguments $[10,11]$.

The distortion of the flow field due to the finite size of the system (and the associated use of PBCs) not only affects the diffusion coefficient of particles, but in principle all dynamical properties. In particular, hydrodynamic flows in an unbounded fluid result in long-time tails of correlation functions, e.g., as $t^{-3 / 2}$ for the velocity autocorrelation function (VACF) in three dimensions [12,13]. Such long-time tails have been reported in molecular simulations for the VACF since the pioneering work of Ref. [14] (see, e.g., [15]) as well as in purely hydrodynamic lattice simulations for the VACF or other correlation functions [16-19]. They have further been observed experimentally on colloidal particles [20-22]. Their trapping in a harmonic potential by optical tweezers modifies the decay of the VACF which remains, however, algebraic [23]. Such slow hydrodynamic modes also manifest themselves in the non-Markovian dynamics of solutes, which includes a deterministic component of the force exerted by the suspending fluid, well described for colloidal spheres by the Basset-Boussinesq force [24,25]. Simulations displaying such a hydrodynamic memory, either on a coarse-grained [20] or molecular [26] scale, may therefore suffer from artifacts associated with the use of PBCs, at least on long-time scales. This was already recognized by Alder and Wainwright in their seminal paper where they reported their results "up to the time where serious interference between neighboring periodically repeated systems is indicated" [27].

Here we address this issue of finite-size effects on the transient regime by revisiting the above hydrodynamic approach. We investigate the transient response to a singular perturbation of the fluid, previously considered to predict the steady-state mobility $[2,28]$. More precisely, we determine numerically the time-dependent Green's function for the Navier-Stokes (NS) equation using lattice-Boltzmann (LB) simulations [29]. We validate this approach in the steady state by comparison with known results, before turning to the transient hydrodynamic response. We show that the multiple features of these finite-size effects can be rationalized analytically by considering the decay of the relevant hydrodynamic modes.

The dynamics of an incompressible fluid of mass density $\rho_{m}$ and shear viscosity $\eta$ can be described by the mass conservation $\partial_{t} \rho_{m}+\rho_{m} \nabla \cdot \mathbf{v}=0$ and NS equation

$$
\rho_{m} \frac{\partial \mathbf{v}}{\partial t}+\rho_{m}(\mathbf{v} \cdot \nabla) \mathbf{v}=\eta \nabla^{2} \mathbf{v}-\nabla p+\mathbf{f},
$$


where $\mathbf{v}$ is the velocity field, $p$ is the pressure, and $\mathbf{f}$ is a force density. In the limit of small Reynolds number $(\mathrm{Re}=$ $\frac{\left\|\rho_{m}(\mathbf{v} \cdot \nabla) \mathbf{v}\right\|}{\left\|\eta \nabla^{2} \mathbf{v}\right\|} \sim \frac{u L}{v}$ with $u$ and $L$ the typical velocity and length, and $v=\eta / \rho_{m}$ the kinematic viscosity), both tensors in Eq. (1) can be obtained by determining the Green's function for the Stokes equation. This corresponds to a vanishing left-hand side in Eq. (2) and a perturbation

$$
\mathbf{f}\left(\mathbf{r}^{\prime}\right)=\left[\delta\left(\mathbf{r}^{\prime}-\mathbf{r}\right)-\frac{1}{V}\right] \mathbf{F},
$$

with $\delta$ the Dirac distribution, $\mathbf{F}$ a force, and $V$ the volume of the system, i.e., a singular point force at $\mathbf{r}$ and a uniform compensating background. The mobility tensor then follows from the steady-state velocity as $\mathbf{v}\left(\mathbf{r}^{\prime}\right)=\mathbb{T}\left(\mathbf{r}^{\prime}, \mathbf{r}\right) \cdot \mathbf{F}$. Note that the limit in Eq. (1) corresponds to $\mathbf{r}^{\prime} \rightarrow \mathbf{r}$. The result for the unbounded case is the well-known Oseen tensor $\mathbb{T}_{\infty}(\mathbf{r})=\frac{1}{8 \pi \eta r}\left(\mathbb{1}+\frac{\mathbf{r r}}{r^{2}}\right)$, while under PBC it is more conveniently expressed in Fourier space [2].

Similarly, the full dynamical response can be obtained by considering a perturbation of the form $\mathbf{f}\left(\mathbf{r}^{\prime}\right) \Theta(t)$, where $\Theta(t)$ is the Heaviside function and the spatial dependence is given by Eq. (3), applied on a fluid initially at rest. The Green's function for the time-dependent NS equation, which corresponds to a perturbation $\mathbf{f}\left(\mathbf{r}^{\prime}\right) \delta(t)$, is obtained as the time derivative of the solution $\mathbf{v}\left(r^{\prime}, t\right)$. In the limit $\operatorname{Re} \ll 1$, the response to $\mathbf{f}\left(\mathbf{r}^{\prime}\right) \Theta(t)$ converges at long times toward the stationary field corresponding to the mobility tensor.

The transient hydrodynamic regime, as quantified by the Green's function, is also related to the equilibrium fluctuations of the velocity field. Using linear response theory [13], it is easy to show that the average velocity $v$ (canonical average over initial configurations) in the direction of the force at the position where it is applied, evolves as $\frac{d}{d t}\langle v(\mathbf{r}, t)\rangle=$ $\frac{1}{k_{B} T} \int d \mathbf{r}^{\prime}\left\langle v(\mathbf{r}, t) v\left(\mathbf{r}^{\prime}, 0\right)\right\rangle f\left(\mathbf{r}^{\prime}, t\right)$. This simplifies for the perturbation considered in Eq. (3), since the total applied force vanishes and so does the total momentum $\int d \mathbf{r}^{\prime} v\left(\mathbf{r}^{\prime}, 0\right)$. One can finally express the velocity autocorrelation of the local velocity field (LVACF) as

$$
Z(t) \equiv\langle v(\mathbf{r}, t) v(\mathbf{r}, 0)\rangle=\frac{k_{B} T}{F} \frac{d\langle v(\mathbf{r}, t)\rangle}{d t},
$$

where $\langle v(\mathbf{r}, t)\rangle$ is the response to the perturbation Eq. (3). This expression, although similar to the one for the velocity of a particle under a constant force $F$, has in fact a very different meaning: Here a perturbation is applied at a fixed position $\mathbf{r}$ (together with the compensating background) and the fluid velocity is followed at that position. Integrating between 0 and infinity, one obtains the steady-state velocity $v_{\infty}(\mathbf{r})=\lim _{t \rightarrow \infty}\langle v(\mathbf{r}, t)\rangle=\frac{F}{k_{B} T} \int_{0}^{\infty}\langle v(\mathbf{r}, t) v(\mathbf{r}, 0)\rangle d t$. This relation is analogous to Einstein's relation for the mobility of a particle, $\mu=v / F=D / k_{B} T$, with the diffusion coefficient $D=\int_{0}^{\infty} Z(t) d t$. In the following we will therefore refer to the integral of the LVACF as the diffusion coefficient.

Here we use LB simulations [29] to solve the above hydrodynamic problem, i.e., the NS equation for a fluid initially at rest on which the perturbation Eq. (3) is applied. In a nutshell, the LB method evolves the one-particle velocity distribution $f(\mathbf{r}, \mathbf{c}, t)$ from which the hydrodynamic moments (density, momentum, stress tensor) can be computed. In (a)

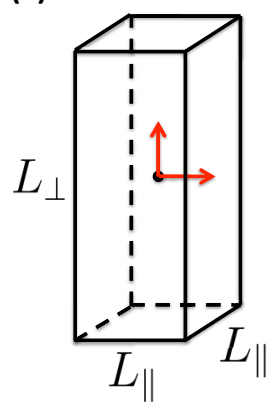

(b)

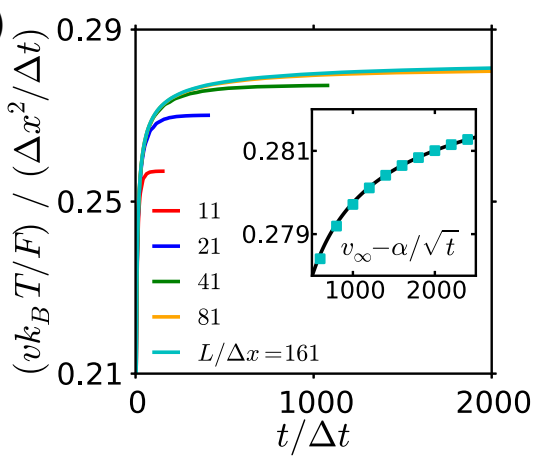

FIG. 1. (a) A bulk fluid in an orthorhombic cell with one length different from the other two is submitted to a perturbation Eq. (3) which corresponds to a singular point force (in one of the two relevant directions indicated by red arrows) and a uniform compensating background. Both elongated $\left(L_{\perp}>L_{\|}\right.$, as shown) and flat $\left(L_{\perp}<L_{\|}\right)$ boxes are considered. (b) Velocity at point $\mathbf{r}$ where the perturbation is applied, as a function of time, from lattice-Boltzmann simulations with various cubic boxes of size $L=L_{\perp, \|}$ (increasing from bottom to top). The inset shows the scaling at long times used to extrapolate the steady-state velocity, for the largest system.

practice, a kinetic equation is discretized in space (lattice spacing $\Delta x$ ) and time (time step $\Delta t$ ) and so are the velocities, which belong to a finite set $\left\{\mathbf{c}_{i}\right\}$ (here we use the D3Q19 lattice). The populations $f_{i}(\mathbf{r}, t) \equiv f\left(\mathbf{r}, \mathbf{c}_{i}, t\right)$ are updated as follows:

$$
\begin{aligned}
f_{i}(\mathbf{r} & \left.+\mathbf{c}_{i} \Delta t, t+\Delta t\right) \\
& =f_{i}(\mathbf{r}, t)-\frac{\Delta t}{\tau}\left[f_{i}(\mathbf{r}, t)-f_{i}^{\mathrm{eq}}(\mathbf{r}, t)\right]+F_{i}^{\mathrm{ext}}(\mathbf{r}, t),
\end{aligned}
$$

where $f_{i}^{\text {eq }}(\mathbf{r}, t)$ corresponds to the local Maxwell-Boltzmann equilibrium with density $\rho(\mathbf{r}, t)=\sum_{i} f_{i}(\mathbf{r}, t)$ and momentum $\rho \mathbf{v}(\mathbf{r}, t)=\sum_{i} f_{i}(\mathbf{r}, t) \mathbf{c}_{i}$, expanded to second order in the velocity to minimize discretization effects resulting, e.g., in numerical viscosity. The relaxation time $\tau$ controls the fluid viscosity. Here we use $\tau=\Delta t$, which results in a kinematic viscosity $v=\frac{c_{s}^{2} \Delta t}{2}=\frac{1}{6} \frac{\Delta x^{2}}{\Delta t}$ since for the D3Q19 lattice the speed of sound is $c_{s}=\frac{1}{\sqrt{3}} \frac{\Delta x}{\Delta t}$ and $F_{i}^{\text {ext }}$ accounts for the external force acting on the fluid [29]. We perform simulations for orthorhombic cells with one length $\left(L_{\perp}\right)$ different from the other two $\left(L_{\|}\right)$, as illustrated in Fig. 1(a), in order to analyze the effect of both the system size and shape.

Starting from $f_{i}^{\text {eq }}$ for a uniform fluid at rest, we apply the singular perturbation to a single node (an extension to arbitrary singular forces, including off-lattice, has been proposed in Ref. [30]) with the compensating background everywhere and monitor the velocity on that node [see Fig. 1(b)]. The same force $F$ is applied for all systems $\left(10^{-4}\right.$ lattice units to ensure that the Mach number is always small: $\mathrm{Ma}=\frac{v}{c_{s}}<10^{-3}$ ) whose sizes are chosen to remain in the limit of small Knudsen numbers $\left(\mathrm{Kn} \sim \frac{\mathrm{Ma}}{\operatorname{Re}}=\frac{v}{c_{s} L} \sim \frac{\Delta x}{L}<0.1\right)$, with $\operatorname{Re}$ at most $\mathcal{O}(1)$.

As a validation of this approach for the computation of hydrodynamic Green's functions, we first describe the results for the diffusion coefficient, obtained from the steady-state velocity as $D=k_{B} T v_{\infty}(\mathbf{r}) / F$. Here the diffusion tensor is anisotropic and the two independent components $D_{\|, \perp}$ are determined by applying the perturbation Eq. (3) in the 


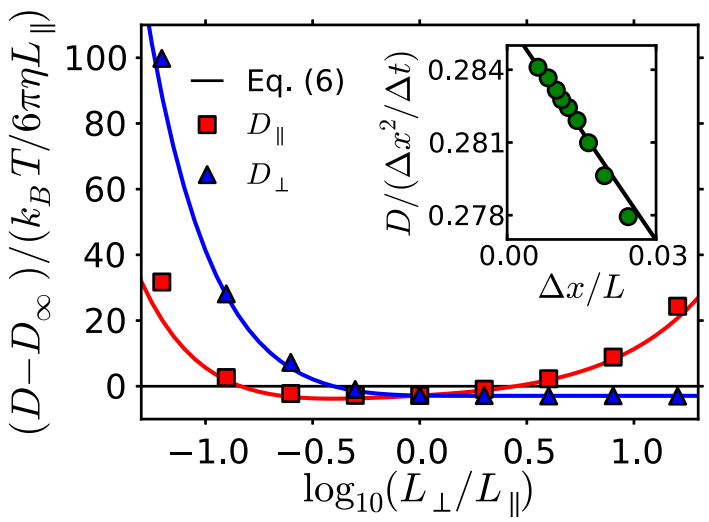

FIG. 2. Scaling functions $h_{\|, \perp}=\left(D_{\|, \perp}-D_{\infty}\right) /\left(k_{B} T / 6 \pi \eta L_{\|}\right)$ defined in Eq. (6) as a function of the aspect ratio $L_{\perp} / L_{\|}$. LatticeBolzmann results (symbols) are compared to analytical results (lines) from Ref. [10]. Note the logarithmic scale on the $x$ axis. Each point corresponds to the slope of a scaling with system size at fixed aspect ratio, as illustrated in the inset for a cubic box $\left(L=L_{\perp, \|}\right)$, where the line again corresponds to Eq. (6).

corresponding directions. Continuum hydrodynamics predicts a scaling with system size [10]:

$$
D_{\|, \perp}=D_{\infty}+\frac{k_{B} T}{6 \pi \eta L_{\|}} h_{\|, \perp}\left(\frac{L_{\perp}}{L_{\|}}\right),
$$

where the two functions $h_{\|, \perp}$ depend only on the aspect ratio $L_{\perp} / L_{\|}$(see Ref. [10]). For the isotropic case $h_{\|, \perp}(1)=-\xi \approx$ -2.837 . The inset of Fig. 2 shows the diffusion coefficient for a cubic box as a function of the size $L_{\perp}=L_{\|}$. For reasons discussed below, the velocity $\langle v(\mathbf{r}, t)\rangle$ converges slowly to its steady-state value, as $v_{\infty}-\alpha / \sqrt{t}$ [see the inset of Fig. 1(b)]. Therefore we used a fit to this expression at long times to determine $v_{\infty}$ for the larger systems.

The LB results are in excellent agreement with the slope expected from Eq. (6), even though some deviations are observed for the smaller box sizes $(\sim 10 \Delta x)$ as expected. The extrapolated value for an infinite system is $D_{\infty} \approx$ $0.286 \Delta x^{2} / \Delta t$. By performing similar size scalings for various aspect ratios (see Table I), we can compute the scaling functions $h_{\|, \perp}$ for both components of the diffusion tensor. The results, shown in the main part of Fig. 2, are also in excellent agreement with Eq. (6). This validates the present approach combining linear response and LB simulations to determine hydrodynamic finite-size effects on the steady-state dynamics.

We now turn to the transient regime. As explained above, the Green's function for the time-dependent NS equation is obtained from the derivative of the response $v(\mathbf{r}, t)$ to the perturbation $\mathbf{f}\left(\mathbf{r}^{\prime}\right) \Theta(t)$. More precisely, we discuss here the LVACF defined by Eq. (4) which is proportional to this Green's function and quantifies the equilibrium hydrodynamic fluctuations. In an unbounded medium, such fluctuations result in the long-time tail of the VACF in simple fluids according to [13]

$$
Z_{\infty}(t)=\frac{2}{3} \frac{k_{B} T}{\rho_{m}}[4 \pi v t]^{-3 / 2} .
$$

TABLE I. The simulated systems correspond to orthorhombic boxes with $L_{x}=L_{y}=L_{\|}$and $L_{z}=L_{\perp}$. For each size ratio $\alpha=$ $L_{\perp} / L_{\|}$, we compute the steady-state velocity as a function of $k_{B} T / 6 \pi \eta L_{\|}$, for a singular force applied either in the $x$ or $z$ direction. The corresponding slopes provide the scaling functions $h_{\|, \perp}$ reported in Fig. 2.

\begin{tabular}{lcccccccccc}
\hline \hline Size ratio $\alpha$ & Lengths & \multicolumn{8}{c}{ Values (in lattice unit $\Delta x$ ) } & \\
\hline $1 / 16$ & $\mathbf{L}_{\|}$ & 175 & 271 & 337 & 429 & & & & \\
& $\mathbf{L}_{\perp}$ & 11 & 17 & 21 & 27 & & & & \\
$1 / 8$ & $\mathbf{L}_{\|}$ & 103 & 119 & 135 & 151 & 169 & & & \\
& $\mathbf{L}_{\perp}$ & 13 & 15 & 17 & 19 & 21 & & & \\
$1 / 4$ & $\mathbf{L}_{\|}$ & 85 & 115 & 125 & 155 & 165 & 175 & & \\
& $\mathbf{L}_{\perp}$ & 21 & 29 & 31 & 39 & 41 & 43 & & & \\
$1 / 2$ & $\mathbf{L}_{\|}$ & 31 & 41 & 51 & 61 & 71 & 101 & & & \\
& $\mathbf{L}_{\perp}$ & 15 & 21 & 25 & 31 & 35 & 51 & & & \\
1 & $\mathbf{L}_{\|}$ & 11 & 21 & 41 & 81 & 161 & & & & \\
& $\mathbf{L}_{\perp}$ & 11 & 21 & 41 & 81 & 161 & & & & \\
2 & $\mathbf{L}_{\|}$ & 11 & 17 & 23 & 29 & 35 & 41 & 47 & 53 & 59 \\
& $\mathbf{L}_{\perp}$ & 22 & 35 & 47 & 59 & 71 & 83 & 95 & 107 & 119 \\
4 & $\mathbf{L}_{\|}$ & 11 & 17 & 23 & 29 & 35 & 41 & 47 & & \\
& $\mathbf{L}_{\perp}$ & 45 & 69 & 93 & 117 & 141 & 165 & 189 & & \\
8 & $\mathbf{L}_{\|}$ & 11 & 17 & 23 & 29 & 35 & & & & \\
& $\mathbf{L}_{\perp}$ & 89 & 137 & 185 & 233 & 281 & & & \\
16 & $\mathbf{L}_{\|}$ & 11 & 17 & 23 & 29 & 35 & & & \\
& $\mathbf{L}_{\perp}$ & 175 & 273 & 369 & 465 & 561 & & & \\
\hline \hline
\end{tabular}

Mode-coupling theory predicts, in fact, a scaling with $D+v$ instead of $v$, but here the Green's function is not associated with the diffusion of a tagged particle, so that $D$ is not involved.

Figure 3 reports the LVACF computed from Eq. (4) using the present LB approach, for various cubic boxes of size $L$. For the larger systems, the simulation results coincide

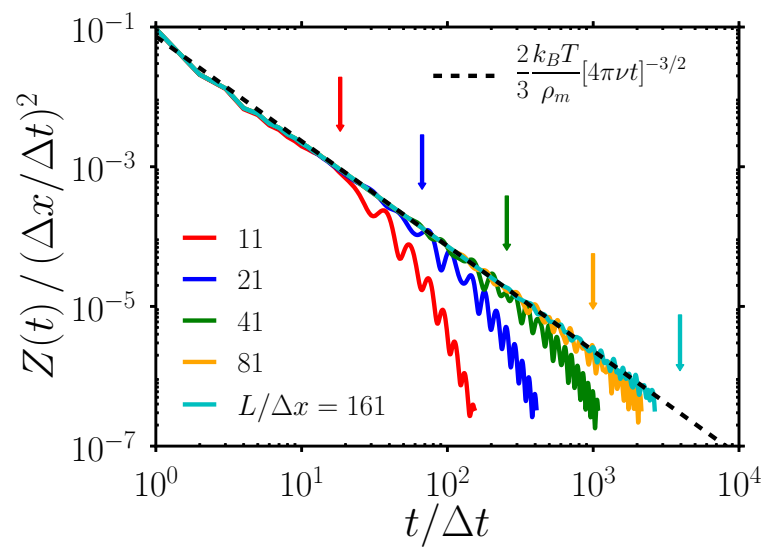

FIG. 3. LVACF computed from lattice-Boltzmann simulations in a cubic cell, for various cell sizes $L / \Delta x$ (increasing from left to right). The double logarithmic scale underlines the algebraic decay expected from hydrodynamics in an unbounded fluid, Eq. (7). The finite size results in a crossover to an exponential decay, analyzed in further detail in Fig. 4. The arrows indicate the diffusion time for the slowest mode, $\tau_{L}=1 / \nu k_{L}^{2}$, with $k_{L}=2 \pi / L$, which corresponds to the exponential decay rate and is also typical of the crossover between the algebraic and exponential regimes. 
exactly with the hydrodynamic scaling Eq. (7) over several orders of magnitude, without any adjustable parameter. This scaling, together with Eq. (4), justifies a posteriori the fit of the velocity as $v_{\infty}-\alpha / \sqrt{t}$ to extrapolate the steady-state value. However, we observe a crossover from the algebraic decay to an exponential regime (and oscillations discussed in more detail below), with a crossover time that decreases with decreasing $L$.

The algebraic decay Eq. (7) arises from the superposition of an infinite number of modes (corresponding to the hydrodynamic limit of vanishing wave numbers $k \rightarrow 0$ ) for momentum diffusion, which in Fourier space decay as $\sim e^{-v k^{2} t}$. The exponential decay therefore results from the cutoff at low wave numbers introduced by the PBC, with the slowest mode corresponding to $k_{L}=2 \pi / L$ and a characteristic time $\tau_{L}=1 / \nu k_{L}^{2}$. The vertical arrows in Fig. 3, which indicate this time, show that it is also typical of the crossover from algebraic to exponential decay $A_{L} e^{-v k_{L}^{2} t}$ of the LVACF. The scaling of the crossover time $\tau_{L}$ is consistent with that reported for the velocity decay of a particle submitted to an impulsive force in LB simulations with fixed system size and varying viscosity [30]. The prefactor $A_{L}$ can be roughly estimated by assuming the continuity between the two regimes at $t=\tau_{L}$. Using Eq. (7), this results in

$$
A_{L}=\frac{2 e}{3[4 \pi]^{3 / 2}} \frac{k_{B} T}{\rho_{m}} k_{L}^{3}
$$

Another striking feature in Fig. 3 is the presence of oscillations, with a frequency which depends on the size of the simulation box. This is clearly another finite-size effect, which can be understood in terms of the slight compressibility of the fluid. Indeed, in the LB method the fluid is only quasi-incompressible. In such a case, while the transverse mode decays as $\sim e^{-v k^{2} t}$ (as for an incompressible fluid), the longitudinal modes follow a dispersion relation which can be obtained by linearizing the mass conservation and compressible NS equation, for an isothermal perturbation of the form $e^{i(\omega t-\mathbf{k} \cdot \mathbf{r})}$. Since the equation of state of the LB fluid is that of an ideal gas $\left(p=\rho k_{B} T=\rho_{m} c_{s}^{2}\right)$, one obtains $(i \omega)^{2}+i \omega k^{2}\left(\frac{4}{3} v+v^{\prime}\right)+c_{s}^{2} k^{2}=0$, with $v^{\prime}=\zeta / \rho_{m}$ the kinematic bulk viscosity. In the case of the D3Q19 lattice, for which $v^{\prime}=\frac{2}{3} v$, the solutions are of the form $i \omega=$ $-v k^{2} \pm i k c_{s} \sqrt{1-v^{2} k^{2} / c_{s}^{2}} \sim-v k^{2} \pm i k c_{s} \quad\left(\right.$ for $\left.k \ll c_{s} / v\right)$, i.e., attenuated sound waves. Such a dispersion relation had already been considered for the LB simulation of acoustic waves (see, e.g., [31-33]).

For periodic systems, the slowest modes correspond to $k_{L}=$ $2 \pi / L$ and longitudinal modes decay as $\sim e^{-v k_{L}^{2} t} \cos \omega_{L} t$, with a frequency $\omega_{L}=k_{L} c_{s} \sqrt{1-v^{2} k_{L}^{2} / c_{s}^{2}} \sim k_{L} c_{s}$. Figure 4 reports the LVACF normalized by the exponential decay $A_{L} e^{-v k_{L}^{2} t}$, as a function of the rescaled time $\omega_{L} t$, for various system sizes spanning more than one order of magnitude. It clearly shows that the above analysis captures all the main features of the finite-size effects on the transient hydrodynamic response: (1) the rate of the exponential decay, since at long times the curves oscillate around a plateau; (2) the order of magnitude $A_{L}$ of the exponential regime, since the value of the plateau is the same

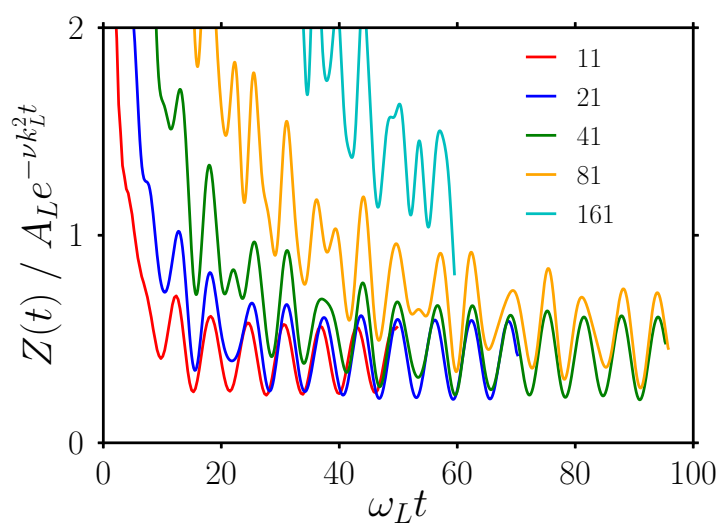

FIG. 4. LVACF computed from lattice-Boltzmann simulations in a cubic cell, for various cell sizes $L / \Delta x$ (increasing from left to right), normalized by the expected exponential scaling at long times $A_{L} e^{-v k_{L}^{2} t}$ with $k_{L}=2 \pi / L$ and $A_{L}$ given by Eq. (8), as a function of time rescaled by the frequency $\omega_{L}=k c_{s} \sqrt{1-v^{2} k^{2} / c_{s}^{2}}$. The oscillations are due to the small compressibility of the LB fluid, which results in damped acoustic waves. For the larger systems the contribution of faster modes $n k_{L}$ is still visible on the time scale of the simulations.

for all system sizes; and (3) the frequency of the oscillations, which are in phase after rescaling by $\omega_{L}$. While only the slowest mode contributes to the oscillations for the smallest system $(L=11 \Delta x)$, others are increasingly visible in this time range as the system size increases. Indeed, the other modes $n k_{L}$ decay as $\sim e^{-v n^{2} k_{L}^{2} t}=e^{-\left(v n^{2} k_{L}^{2} / \omega_{L}\right) \omega_{L} t}$, i.e., $\frac{v n^{2} k_{L}^{2}}{\omega_{L}}=n^{2} \frac{v k_{L}}{c_{s}}$ times faster-a difference which decreases with increasing $\stackrel{\omega_{L}}{L}$. Using more elaborate LB schemes for compressible thermal flows [34] or any other hydrodynamic simulation algorithm (or even the continuous Boltzmann equation from which the LB algorithm follows), will also result in the crossover to the exponential regime, which is an inevitable consequence of the finite size of the system and the associated PBC. It should also manifest itself with strictly incompressible flow simulations, even though we do not expect oscillations in the $\mathrm{VACF}$ in that case.

Overall, the present work shows that it is possible to rationalize all finite-size effects in terms of the cutoff of hydrodynamic modes at small wave numbers introduced by the use of PBC. Coming back to Alder and Wainwright's quote [27], the time where neighboring periodically repeated systems seriously interfere corresponds to momentum diffusion for the slowest mode, $\tau_{L}=1 / \nu k_{L}^{2}$. It is crucial for the setup and analysis of molecular simulations to control these finite-size effects, which can be efficiently computed from the present approach combining linear response and LB simulations. In turn, such an analysis is useful to extrapolate the macroscopic limit without actually performing the simulations for too large systems. One could exploit these effects further to determine material properties, not only the viscosity from the slope of the diffusion coefficient vs inverse box size (as for water in first-principles molecular dynamics simulations [35]), but also, e.g., the speed of sound from the oscillation frequency of the LVACF, as shown here. 
The systematic finite-size analysis of the transient response could also be extended to other situations. For example, the long-time decay of the VACF under confinement or near a boundary, in an otherwise unbounded fluid, scales as $t^{-5 / 2}$ instead of $t^{-3 / 2}$ in the bulk [36,37], but PBCs in the directions parallel to the interface will also result in deviations from the algebraic decay. Similarly, the diffusion coefficient of lipids and carbon nanotubes embedded in a membrane diverges logarithmically with system size [11] and one should also observe the impact of PBC on the transient dynamics. This may also prove important for extracting from finite-size simulations other dynamical properties for which hydrodynamics play an important role, such as memory kernels [26].

\section{ACKNOWLEDGMENTS}

The authors are grateful to Jean-Pierre Hansen and Lydéric Bocquet for useful discussions. A.J.A. and B.R. acknowledge financial support from the French Agence Nationale de la Recherche (ANR) under Grant No. ANR-15-CE09-0013.
[1] B. Dünweg and K. Kremer, Molecular dynamics simulation of a polymer chain in solution, J. Chem. Phys. 99, 6983 (1993).

[2] I.-C. Yeh and G. Hummer, System-size dependence of diffusion coefficients and viscosities from molecular dynamics simulations with periodic boundary conditions, J. Phys. Chem. B 108, 15873 (2004).

[3] M. Fushiki, System size dependence of the diffusion coefficient in a simple liquid, Phys. Rev. E 68, 021203 (2003).

[4] S. Tazi, A. Boţan, M. Salanne, V. Marry, P. Turq, and B. Rotenberg, Diffusion coefficient and shear viscosity of rigid water models, J. Phys.: Condens. Matter 24, 284117 (2012).

[5] D. Rozmanov and P. G. Kusalik, Transport coefficients of the TIP4P-2005 water model, J. Chem. Phys. 136, 044507 (2012).

[6] S. Gabl, C. Schröder, and O. Steinhauser, Computational studies of ionic liquids: Size does matter and time too, J. Chem. Phys. 137, 094501 (2012).

[7] S. P. Singh, C.-C. Huang, E. Westphal, G. Gompper, and R. G. Winkler, Hydrodynamic correlations and diffusion coefficient of star polymers in solution, J. Chem. Phys. 141, 084901 (2014).

[8] G. Kikugawa, S. Ando, J. Suzuki, Y. Naruke, T. Nakano, and T. Ohara, Effect of the computational domain size and shape on the self-diffusion coefficient in a Lennard-Jones liquid, J. Chem. Phys. 142, 024503 (2015).

[9] G. Kikugawa, T. Nakano, and T. Ohara, Hydrodynamic consideration of the finite size effect on the self-diffusion coefficient in a periodic rectangular parallelepiped system, J. Chem. Phys. 143, 024507 (2015).

[10] A. Botan, V. Marry, and B. Rotenberg, Diffusion in bulk liquids: Finite-size effects in anisotropic systems, Mol. Phys. 113, 2674 (2015).

[11] M. Vögele and G. Hummer, Divergent diffusion coefficients in simulations of fluids and lipid membranes, J. Phys. Chem. B 120, 8722 (2016).

[12] M. H. Ernst, E. H. Hauge, and J. M. J. van Leeuwen, Asymptotic time behavior of correlation functions. I. Kinetic terms, Phys. Rev. A 4, 2055 (1971).

[13] J.-P. Hansen and I. R. McDonald, Theory of Simple Liquids, 4th ed. (Academic Press, New York, 2013).

[14] B. J. Alder and T. E. Wainwright, Velocity Autocorrelations for Hard Spheres, Phys. Rev. Lett. 18, 988 (1967).

[15] D. Levesque and W. T. Ashurst, Long-Time Behavior of the Velocity Autocorrelation Function for a Fluid of Soft Repulsive Particles, Phys. Rev. Lett. 33, 277 (1974).

[16] M. A. van der Hoef and D. Frenkel, Long-time tails of the velocity autocorrelation function in two- and three-dimensional lattice-gas cellular automata: A test of mode-coupling theory, Phys. Rev. A 41, 4277 (1990).

[17] C. P. Lowe, D. Frenkel, and A. J. Masters, Long-time tails in angular momentum correlations, J. Chem. Phys. 103, 1582 (1995).

[18] M. A. van der Hoef and D. Frenkel, Computer simulations of long-time tails: What's new? Transp. Theory Stat. Phys. 24, 1227 (1995).

[19] C. P. Lowe and D. Frenkel, The super long-time decay of velocity fluctuations in a two-dimensional fluid, Physica A: Stat. Mech. Appl. 220, 251 (1995).

[20] T. Franosch, M. Grimm, M. Belushkin, F. M. Mor, G. Foffi, L. Forró, and S. Jeney, Resonances arising from hydrodynamic memory in Brownian motion, Nature (London) 478, 85 (2011).

[21] T. Li and M. G. Raizen, Brownian motion at short time scales, Ann. Phys. (NY) 525, 281 (2013).

[22] S. Kheifets, A. Simha, K. Melin, T. Li, and M. G. Raizen, Observation of Brownian motion in liquids at short times: Instantaneous velocity and memory loss, Science 343, 1493 (2014).

[23] H. J. H. Clercx and P. P. J. M. Schram, Brownian particles in shear flow and harmonic potentials: A study of long-time tails, Phys. Rev. A 46, 1942 (1992).

[24] J Boussinesq, Théorie Analytique de la Chaleur (GauthierVillars, Paris, France, 1903), Vol. 2.

[25] T. S. Chow and J. J. Hermans, Effect of inertia on the Brownian motion of rigid particles in a viscous fluid, J. Chem. Phys. 56, 3150 (1972).

[26] D. Lesnicki, R. Vuilleumier, A. Carof, and B. Rotenberg, Molecular Hydrodynamics from Memory Kernels, Phys. Rev. Lett. 116, 147804 (2016).

[27] B. J. Alder and T. E. Wainwright, Decay of the velocity autocorrelation function, Phys. Rev. A 1, 18 (1970).

[28] H. Hasimoto, On the periodic fundamental solutions of the Stokes equations and their application to viscous flow past a cubic array of spheres, J. Fluid Mech. 5, 317 (1959).

[29] S. Succi, The Lattice Boltzmann Equation for Fluid Dynamics and Beyond (Oxford University Press, New York, 2001).

[30] R. W. Nash, R. Adhikari, and M. E. Cates, Singular forces and pointlike colloids in lattice Boltzmann hydrodynamics, Phys. Rev. E 77, 026709 (2008).

[31] P. J. Dellar, Bulk and shear viscosities in lattice Boltzmann equations, Phys. Rev. E 64, 031203 (2001). 
[32] Y. Li and X. Shan, Lattice Boltzmann method for adiabatic acoustics, Philos. Trans. R. Soc. London, Ser. A 369, 2371 (2011).

[33] E. M. Viggen, Viscously damped acoustic waves with the lattice Boltzmann method, Philos. Trans. R. Soc. London, Ser. A 369, 2246 (2011).

[34] N. I. Prasianakis and I. V. Karlin, Lattice Boltzmann method for simulation of compressible flows on standard lattices, Phys. Rev. E 78, 016704 (2008).
[35] T. D. Kühne, M. Krack, and M. Parrinello, Static and dynamical properties of liquid water from first principles by a novel Car-Parrinello-like approach, J. Chem. Theory Comput. 5, 235 (2009).

[36] M. H. J. Hagen, I. Pagonabarraga, C. P. Lowe, and D. Frenkel, Algebraic Decay of Velocity Fluctuations in a Confined Fluid, Phys. Rev. Lett. 78, 3785 (1997).

[37] K. Huang and I. Szlufarska, Effect of interfaces on the nearby Brownian motion, Nat. Commun. 6, 8558 (2015). 